\title{
APPROXIMATE SOLUTIONS OF FOURTH-ORDER FRACTIONAL INTEGRO-DIFFERENTIAL EQUATIONS
}

\author{
S.M. Atshan, A.A. Hamoud
}

ABstRACT. In this paper, linear boundary value problems for fourth-order Caputo fractional Volterra integro-differential equations are solved by variational iteration method and homotopy perturbation method. The solutions of the problems are derived by infinite convergent series which are easily computable and then graphical representation shows that both methods are most effective and convenient one to solve linear boundary value problems for fourth-order fractional integro-differential equations. In order to show the efficiency of the presented methods, we compare our results obtained with the exact results.

2010 Mathematics Subject Classification: 35A15, 26A33, 65H20, 45J05

Keywords: Variational iteration method, Homotopy perturbation method, Caputo fractional Volterra integro-differential equation, Approximate solution.

\section{INTRODUCTION}

In recent years various numerical and analytical methods have been applied for the approximate solutions of fractional integro-differential equations. He $[7,8,9,10]$ was the first to propose the Variational Iteration Method (VIM) and Homotopy Perturbation Method (HPM) for finding the solutions of linear and nonlinear problems. VIM is based on Lagrange multiplier and HPM is a coupling of the traditional perturbation method and homotopy in topology. These methods have been successfully applied by many authors $[1,2,13,15,16,22,23]$ for finding the analytical approximate solutions as well as numerical approximate solutions.

The main objective of this paper is to extend the analysis of VIM and HPM to construct the approximate solutions of the following linear boundary value problems for Caputo fractional Volterra integro-differential equations

$$
{ }^{c} D^{\alpha} u(x)=\gamma u(x)+g(x)+\int_{0}^{x} k(x, t) u(t) d t, \quad 0<x<b,
$$


with the boundary conditions

$$
\begin{aligned}
& u(0)=\gamma_{0}, \quad u^{\prime \prime}(0)=\gamma_{2}, \\
& u(b)=\eta_{0}, \quad u^{\prime \prime}(b)=\eta_{2},
\end{aligned}
$$

where ${ }^{c} D^{\alpha}$ is the Caputo's fractional derivative, $3<\alpha \leq 4$, and $u: J \longrightarrow \mathbb{R}$, where $J=[0, b]$ is the continuous function which has to be determined, $g: J \longrightarrow \mathbb{R}$ and $k: J \times J \longrightarrow \mathbb{R}$ are continuous functions.

The fractional integro-differential equations have attracted much more interest of mathematicians and physicists which provides an efficiency for the description of many practical dynamical arising in engineering and scientific disciplines such as, physics, biology, electrochemistry, chemistry, economy, electromagnetic, control theory and viscoelasticity $[12,14,16,17,18]$. Recently, many authors focus on the development of numerical and analytical techniques for integro-differential equations. For instance, the Homotopy perturbation method [5], the variational iteration method [11], the combined modified Laplace with Adomian decomposition method $[6,12,13]$, the homotopy-perturbation method [1], Taylor polynomials [3, 20] and Tau method [4], and the references therein.

The main objective of the present paper is to study the behavior of the solution that can be formally determined by approximated methods as the variational iteration method and homotopy perturbation method.

The rest of the paper is organized as follows: In Section 2, some preliminaries and basic definitions related to fractional calculus are recalled. In Section 3, a short review of the homotopy perturbation technique. In Section 4, a short review of the variational iteration technique. In Section 5, variational iteration method and homotopy perturbation method are constructed for solving Caputo fractional Volterra integro-differential equations. In Section 6, an example is presented to illustrate the accuracy of these methods. Finally, we will give a report on our paper and a brief conclusion are given in Section 7 .

\section{Preliminaries}

The mathematical definitions of fractional derivative and fractional integration are the subject of several different approaches. The most frequently used definitions of the fractional calculus involves the Riemann-Liouville fractional derivative, Caputo derivative $[1,21]$.

Definition 2.1. The Riemann Liouville fractional integral of order $\alpha>0$ of a 
function $f \in C(0, \infty)$ is defined as

$$
\begin{aligned}
J^{\alpha} f(x) & =\frac{1}{\Gamma(\alpha)} \int_{0}^{x}(x-t)^{\alpha-1} f(t) d t, \quad x>0, \quad \alpha \in \mathbb{R}^{+}, \\
J^{0} f(x) & =f(x),
\end{aligned}
$$

where $\mathbb{R}^{+}$is the set of positive real numbers.

Definition 2.2. The fractional derivative of $f(x)$ in the Caputo sense is defined by

$$
\begin{aligned}
{ }^{c} D_{x}^{\alpha} f(x) & =J^{m-\alpha} D^{m} f(x) \\
& = \begin{cases}\frac{1}{\Gamma(m-\alpha)} \int_{0}^{x}(x-t)^{m-\alpha-1} \frac{d^{m} f(t)}{d t^{m}} d t, & m-1<\alpha<m, \\
\frac{d^{m} f(x)}{d x^{m}}, & \alpha=m, \quad m \in N,\end{cases}
\end{aligned}
$$

where the parameter $\alpha$ is the order of the derivative and is allowed to be real or even complex. In this paper, only real and positive $\alpha$ will be considered.

Hence, we have the following properties:

1. $J^{\alpha} J^{v} f=J^{\alpha+v} f, \quad \alpha, v>0$.

2. $J^{\alpha} x^{\beta}=\frac{\Gamma(\beta+1)}{\Gamma(\beta+\alpha+1)} x^{\beta+\alpha}$,

3. $J^{\alpha} D^{\alpha} f(x)=f(x)-\sum_{k=0}^{m-1} f^{(k)}\left(0^{+}\right) \frac{x^{k}}{k !}, \quad x>0, \quad m-1<\alpha \leq m$.

Definition 2.3. The Riemann Liouville fractional derivative of order $\alpha>0$ is normally defined as

$$
D^{\alpha} f(x)=D^{m} J^{m-\alpha} f(x), \quad m-1<\alpha \leq m, \quad m \in \mathbb{N} .
$$

\section{Homotopy Perturbation Method (HPM)}

The homotopy perturbation method first proposed by He $[5,8,9,10]$. To illustrate the basic idea of this method, we consider the following nonlinear differential equation

$$
A(u)-f(r)=0, \quad r \in \Omega,
$$

under the boundary conditions

$$
B\left(u, \frac{\partial u}{\partial n}\right)=0, \quad r \in \Gamma,
$$


S.M. Atshan, A.A. Hamoud - Approximate solutions of fourth-order ...

where $A$ is a general differential operator, $B$ is a boundary operator, $f(r)$ is a known analytic function, $\Gamma$ is the boundary of the domain $\Omega$.

In general, the operator $A$ can be divided into two parts $L$ and $N$, where $L$ is linear, while $N$ is nonlinear. Eq. (3.1) therefore can be rewritten as follows

$$
L(u)+N(u)-f(r)=0 .
$$

By the homotopy technique (Liao 1995) [19]. We construct a homotopy $v(r, p)$ : $\Omega \times[0,1] \longrightarrow \mathbb{R}$ which satisfies

$$
H(v, p)=(1-p)\left[L(v)-L\left(u_{0}\right)\right]+p[A(v)-f(r)]=0, p \in[0,1] .
$$

or

$$
\left.H(v, p)=L(v)-L\left(u_{0}\right)+p L\left(u_{0}\right)\right]+p[N(v)-f(r)]=0,
$$

where $p \in[0,1]$ is an embedding parameter, $u_{0}$ is an initial approximation of Eq.(3.1) which satisfies the boundary conditions. From Eqs.(3.4), (3.5) we have

$$
\begin{aligned}
& H(v, 0)=L(v)-L\left(u_{0}\right)=0 \\
& H(v, 1)=A(v)-f(r)=0 .
\end{aligned}
$$

The changing in the process of $p$ from zero to unity is just that of $v(r, p)$ from $u_{0}(r)$ to $u(r)$. In topology this is called deformation and $L(v)-L\left(u_{0}\right)$, and $A(v)-f(r)$ are called homotopic. Now, assume that the solution of Eqs. (3.4), (3.5) can be expressed as

$$
v=v_{0}+p v_{1}+p^{2} v_{2}+\cdots
$$

The approximate solution of Eq.(3.1) can be obtained by Setting $p=1$.

$$
u=\lim _{p \rightarrow 1} v=v_{0}+v_{1}+v_{2}+\cdots
$$

\section{Variational Iteration Method (VIM)}

We consider the following equation $[7,16,22]$ :

$$
D u+M u+N u=g(x),
$$

where $D$ is a differential operator, $M, N$ represents the nonlinear terms, and $g$ is the source term. The basic character of He's method is the construction of a correction functional for (4.1), which reads 
S.M. Atshan, A.A. Hamoud - Approximate solutions of fourth-order ...

$$
u_{n+1}(x)=u_{n}(x)+\int_{0}^{x} \lambda(s)\left[D u_{n}(s)+M \tilde{u}_{n}(s)+N \tilde{u}_{n}(s)-g(s)\right] d s,
$$

where $\lambda$ is a Lagrange multiplier which can be identified optimally via variational theory [7], $u_{n}$ is the $n^{\text {th }}$ approximate solution, and $\tilde{u}_{n}$ denotes a restricted variation, i.e., $\delta \tilde{u}_{n}=0$. To solve (4.1) by He's VIM, we first determine the Lagrange multiplier $\lambda$ that will be identified optimally via integration by parts. Then the successive approximations $u_{n}(x), n \geq 0$, of the solution $u(x)$ will be readily obtained upon using the obtained Lagrange multiplier and by using any selective function $u_{0}$. The approximation $u_{0}$ may be selected by any function that just satisfies at least the initial and boundary conditions. With determined $\lambda$, then several approximations $u_{n}(x), n \geq 0$, follow immediately. We have the following variational iteration formula

$$
\begin{aligned}
& u_{0}(x) \text { is an arbitrary initial guess, } \\
& u_{n+1}(x)=u_{n}(x)+\int_{0}^{x} \lambda(s)\left[D u_{n}(s)+M u_{n}(s)+N u_{n}(s)-g(s)\right] d s,
\end{aligned}
$$

Now we are applying the integral operator $J$ to both sides of (4.1), and using the given conditions, we obtain

$$
u=R-J[M u]-J[N u],
$$

where the function $R$ represents the terms arising from integrating the source term $g$ and from using the given conditions, all are assumed to be prescribed. we have the following variational iteration formula for (4.4)

$$
\begin{aligned}
& u_{0}(x) \text { is an arbitrary initial guess, } \\
& u_{n+1}(x)=R(x)-J\left[M u_{n}(x)\right]-J\left[N u_{n}(x)\right] .
\end{aligned}
$$

\section{Description of the Some Reliable Methods}

Some powerful methods have been focusing on the development of more advanced and efficient methods for integro-differential equations such as the homotopy perturbation method and variational iteration method $[1,5,12,13,22,23]$. We will describe these methods in this section: 


\subsection{Homotopy Perturbation Method}

To solve the Caputo fractional Volterra integro-differential equation (1.1) by using the homotopy perturbation method, with boundary conditions (1.2), one can construct the following correction functional:

$$
(1-P)^{c} D^{\alpha} u(x)+P\left[{ }^{c} D^{\alpha} u(x)-\gamma u(x)-g(x)-\int_{0}^{x} k(x, t) u(t) d t\right]=0 .
$$

In view of basic assumption of HPM, solution of (1.1) can be expressed as a power series in $P$ :

$$
u(x)={ }^{c} D^{\alpha} u_{0}(x)+P^{c} D^{\alpha} u_{1}(x)+P^{2 c} D^{\alpha} u_{2}(x)+P^{3 c} D^{\alpha} u_{3}(x)+\cdots .
$$

If we put $P \longrightarrow 1$ in (5.2), we get the approximate solution of (1.1)

$$
u(x)={ }^{c} D^{\alpha} u_{0}(x)+{ }^{c} D^{\alpha} u_{1}(x)+{ }^{c} D^{\alpha} u_{2}(x)+{ }^{c} D^{\alpha} u_{3}(x)+\cdots .
$$

Now, we substitute (5.2) into (5.1), then equating the terms with identical power of $P$, we obtain the following series of linear equations:

$$
\begin{aligned}
& P^{0}:{ }^{c} D^{\alpha} u_{0}(x)=0, \\
& P^{1}:{ }^{c} D^{\alpha} u_{1}(x)=g(x)+\gamma u_{0}(x)+\int_{0}^{x} k(x, t) u_{0}(t) d t, \\
& P^{2}:{ }^{c} D^{\alpha} u_{2}(x)=\gamma u_{1}(x)+\int_{0}^{x} k(x, t) u_{1}(t) d t, \\
& P^{3}:{ }^{c} D^{\alpha} u_{3}(x)=\gamma u_{2}(x)+\int_{0}^{x} k(x, t) u_{2}(t) d t,
\end{aligned}
$$

the initial approximation can be chosen in the following way:

$$
u_{0}=\sum_{i=0}^{3} \gamma_{i} \frac{x^{i}}{i !},
$$

where $\gamma_{1}=u^{\prime}(0)$ and $\gamma^{3}=u^{\prime \prime \prime}(0)$ are to be determined by applying suitable boundary conditions (1.2). 


\subsection{Variational Iteration Method}

To solve the Caputo fractional Volterra-Fredholm integro-differential equation (1.1) by using the variational iteration method, with boundary conditions (1.2), one can construct the following correction functional:

$$
\begin{aligned}
u_{k+1}(x)= & u_{k}(x)+J^{\beta}\left[\lambda\left({ }^{c} D^{\alpha} u_{k}(x)-\gamma \tilde{u}_{k}(x)-g(x)-\int_{0}^{x} k(x, t) \tilde{u}_{k}(t) d t\right)\right] \\
= & u_{k}(x)+\frac{1}{\Gamma(\beta)} \int_{0}^{x}(x-s)^{\beta-1} \lambda(s) \\
& \times\left({ }^{c} D^{\alpha} u_{k}(s)-\gamma \tilde{u}_{k}(s)-g(s)-\int_{0}^{s} k(s, t) \tilde{u}_{k}(t) d t\right) d s
\end{aligned}
$$

where $J^{\beta}$ is the Riemann-Liouville fractional integral operator of order $\beta=\alpha-m+1$, $m \in \mathbb{N}, \lambda$ is a general Lagrange multiplier and $\tilde{u}$ denotes restricted variation i.e. $\delta \tilde{u}_{k}=0$. We make some approximation for the identification of an approximate Lagrange multiplier, so the correctional functional (5.6) can be approximately expressed as:

$$
u_{k+1}(x)=u_{k}(x)+\int_{0}^{x} \lambda(s)\left({ }^{c} D^{4} u_{k}(s)-\gamma \tilde{u}_{k}(s)-g(s)-\int_{0}^{s} k(s, t) \tilde{u}_{k}(t) d t\right) d s .
$$

Making the above correction functional stationary, we obtain the following stationary conditions:

$1-\left.\lambda^{\prime \prime \prime}(s)\right|_{x=s}=0,\left.\quad \lambda^{\prime \prime}(s)\right|_{x=s}=0 \quad-\left.\lambda^{\prime}(s)\right|_{x=s}=0,\left.\quad \lambda(s)\right|_{x=s}=0,\left.\quad \lambda^{i v}(s)\right|_{x=s}=$ 0 .

This gives the following Lagrange multiplier

$$
\lambda(s)=\frac{1}{6}(s-x)^{3} .
$$

We obtain the following iteration formula by substitution of (5.7) into functional (5.6),

$$
\begin{aligned}
u_{k+1}(x)= & u_{k}(x)+\frac{1}{6 \Gamma(\alpha-3)} \int_{0}^{x}(x-s)^{\alpha-4}(s-x)^{3} \\
& \times\left({ }^{c} D^{\alpha} u_{k}(s)-\gamma u_{k}(s)-g(s)-\int_{0}^{s} k(s, t) u_{k}(t) d t\right) d s .
\end{aligned}
$$


S.M. Atshan, A.A. Hamoud - Approximate solutions of fourth-order ...

Then,

$$
\begin{aligned}
u_{k+1}(x)= & u_{k}(x)-\frac{(\alpha-3)(\alpha-2)(\alpha-1)}{6 \Gamma(\alpha)} \int_{0}^{x}(x-s)^{\alpha-1} \\
& \times\left({ }^{c} D^{\alpha} u_{k}(s)-\gamma u_{k}(s)-g(s)-\int_{0}^{s} k(s, t) u_{k}(t) d t\right) d s .
\end{aligned}
$$

The initial approximation $u_{0}$ can be chosen by the following way which satisfies initial conditions (1.2):

$$
u_{0}(x)=\gamma_{0}+\gamma_{1} x+\gamma_{2} \frac{x^{2}}{2}+\gamma_{3} \frac{x^{3}}{6}
$$

where $\gamma_{1}=u^{\prime}(0)$ and $\gamma^{3}=u^{\prime \prime \prime}(0)$ are to be determined by applying suitable boundary conditions (1.2). We can obtain the first-order and higher-order approximation by substitution of (5.10) into (5.9).

\section{Applications}

In this section we have applied variational iteration method and homotopy perturbation method to linear Caputo fractional Volterra integro-differential equations.

Example 1. Consider the following linear Caputo fractional Volterra integrodifferential equation:

$$
{ }^{c} D^{\alpha} u(x)=u(x)+\left(1+e^{x}\right) x+3 e^{x}-\int_{0}^{x} u(t) d t, \quad 3<\alpha \leq 4, \quad 0<x<1,
$$

with the boundary conditions

$$
\begin{aligned}
& u(0)=1, \quad u^{\prime \prime}(0)=2, \\
& u(1)=1+e, \quad u^{\prime \prime}(1)=3 e .
\end{aligned}
$$

The exact solution of problem (6.1)-(6.2) for $\alpha=4$ is

$$
u(x)=1+x e^{x} .
$$

According to variational iteration method, the iteration formula (5.9) for Eq.(6.1) can be expressed in the following form: 
S.M. Atshan, A.A. Hamoud - Approximate solutions of fourth-order ...

$$
\begin{aligned}
u_{k+1}(x)= & u_{k}(x)-\frac{(\alpha-3)(\alpha-2)(\alpha-1)}{6 \Gamma(\alpha)} \int_{0}^{x}(x-s)^{\alpha-1} \\
& \times\left({ }^{c} D^{\alpha} u_{k}(s)-u_{k}(x)-\left(1+e^{x}\right) x-3 e^{x}+\int_{0}^{x} u_{k}(t) d t\right) d s .
\end{aligned}
$$

In order to avoid difficult fractional integration, we can take the truncated Taylor expansion for the exponential term in (6.4): e.g., $e^{x} \sim 1+x+x^{2} / 2+x^{3} / 6$ and assume that an initial approximation has the following form which satisfies the initial conditions (6.2):

$$
u_{0}(x)=1+A x+x^{2}+B \frac{x^{3}}{6}
$$

where $A=y^{\prime}(0)$ and $B=u^{\prime \prime \prime}(0)$ are unknowns to be determined.

Now, by iteration formula (6.4), first-order approximation takes the following form:

$$
\begin{aligned}
u_{1}(x)= & u_{0}(x)-\frac{(\alpha-3)(\alpha-2)(\alpha-1)}{6 \Gamma(\alpha)} \int_{0}^{x}(x-s)^{\alpha-1} \\
& \times\left({ }^{c} D^{\alpha} u_{0}(s)-u_{0}(x)-\left(1+e^{x}\right) x-3 e^{x}+\int_{0}^{x} u_{0}(t) d t\right) d s . \\
= & 1+A x+x^{2}+B \frac{x^{3}}{6}-\frac{(\alpha-3)(\alpha-2)(\alpha-1) x^{\alpha}}{6} \\
& \times\left(-\frac{4}{\Gamma(\alpha+1)}-\frac{(4+A) x}{\Gamma(\alpha+2)}+\frac{(A-7) x^{2}}{\Gamma(\alpha+3)}-\frac{(4+B) x^{3}}{\Gamma(\alpha+4)}+\frac{(B-4) x^{4}}{\Gamma(\alpha+5)}\right) .
\end{aligned}
$$

According to homotopy perturbation method, we construct the following homotopy:

$$
{ }^{c} D^{\alpha} u(x)=P\left(u(x)+\left(1+e^{x}\right) x+3 e^{x}-\int_{0}^{x} u(t) d t\right) .
$$

Substitution of (6.6) into (5.4) and then equating the terms with same powers of $P$ yield the following series of linear equations:

$$
\begin{aligned}
P^{0}:{ }^{c} D^{\alpha} u_{0}(x) & =0 \\
P^{1}:{ }^{c} D^{\alpha} u_{1}(x) & =u_{0}(x)+\left(1+e^{x}\right) x+3 e^{x}-\int_{0}^{x} u_{0}(t) d t \\
P^{2}:{ }^{c} D^{\alpha} u_{2}(x) & =u_{1}(x)-\int_{0}^{x} u_{1}(t) d t \\
P^{3}:{ }^{c} D^{\alpha} u_{3}(x) & =u_{2}(x)-\int_{0}^{x} u_{2}(t) d t
\end{aligned}
$$


Applying the operator $J^{\alpha}$ to the above series of linear equations and using initial conditions (6.2), we get:

$$
\begin{aligned}
u_{0}(x)= & 1, \\
u_{1}(x)= & A x+x^{2}+\frac{B}{6} x^{3}+\frac{4 x^{\alpha}}{\Gamma(\alpha+1)}+\frac{4 x^{\alpha+1}}{\Gamma(\alpha+2)}+\frac{4 x^{\alpha+2}}{\Gamma(\alpha+3)}+\frac{4 x^{\alpha+3}}{\Gamma(\alpha+4)}+\frac{4 x^{\alpha+4}}{\Gamma(\alpha+5)}, \\
u_{2}(x)= & A \frac{x^{\alpha+1}}{\Gamma(\alpha+2)}+(2-A) \frac{x^{\alpha+2}}{\Gamma(\alpha+3)}+(B-2) \frac{x^{\alpha+3}}{\Gamma(\alpha+4)}-B \frac{x^{\alpha+4}}{\Gamma(\alpha+5)}+4 \frac{x^{2 \alpha}}{\Gamma(2 \alpha+1)} \\
& +\frac{x^{2 \alpha+2}}{\Gamma(2 \alpha+3)}+\frac{x^{2 \alpha+3}}{\Gamma(2 \alpha+4)}-2 \frac{x^{2 \alpha+4}}{\Gamma(2 \alpha+5)}-4 \frac{x^{2 \alpha+5}}{\Gamma(2 \alpha+6)},
\end{aligned}
$$

where $A$ and $B$ can be determined by imposing boundary conditions.

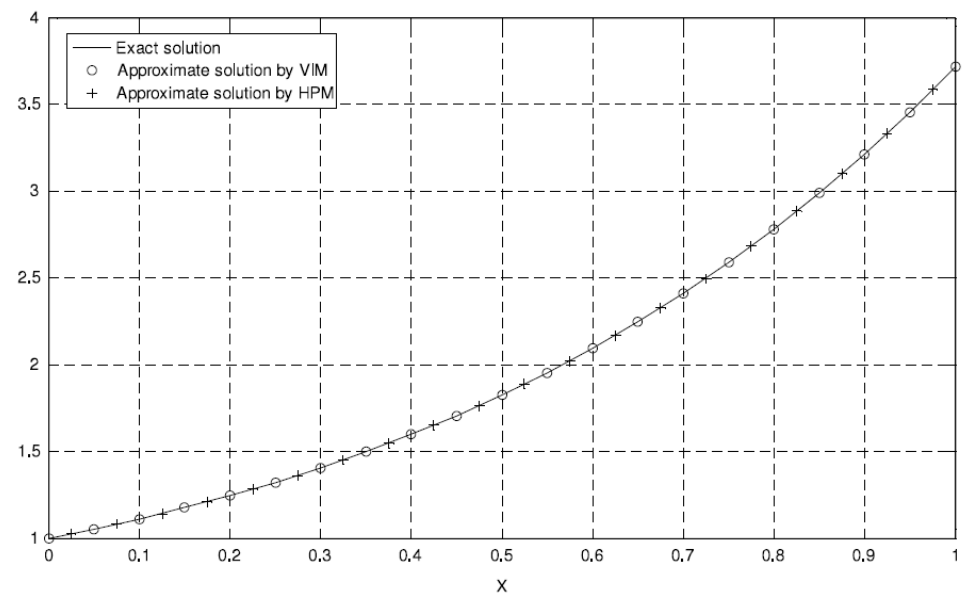

Figure 1: Comparison of approximate solutions obtained by 2-term HPM and firstorder VIM with exact solution at $\alpha=4$.

From Figs. 1. and 2. the approximate solutions are in good agreement with an exact solution of (6.1)-(6.2) at $\alpha=4$ and $\alpha=3.2$. Also it is to be noted that the accuracy can be improved by computing more terms of approximated solutions or by taking more terms in the Taylor expansion for the exponential term. 
S.M. Atshan, A.A. Hamoud - Approximate solutions of fourth-order ...

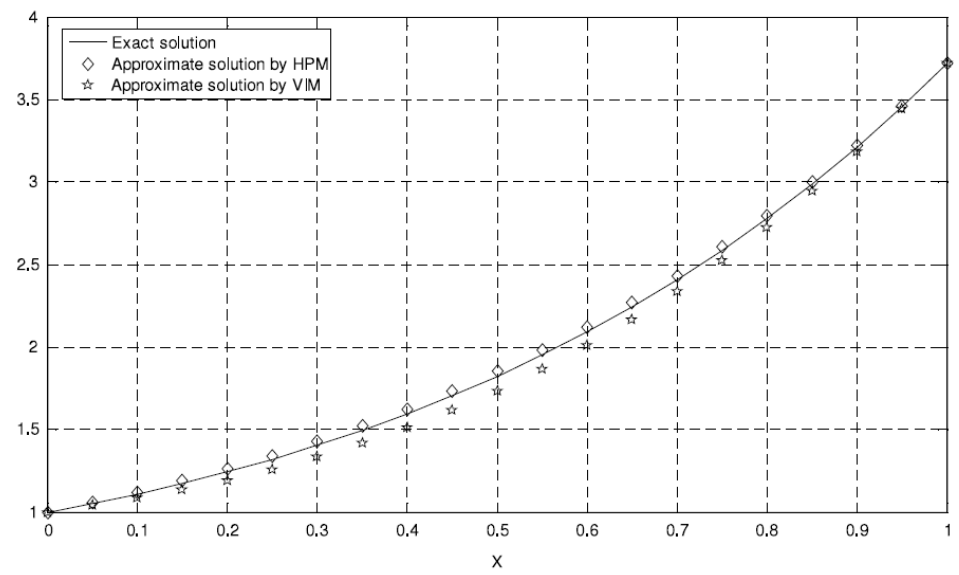

Figure 2: Comparison of approximate solutions obtained by 2-term HPM and firstorder VIM with exact solution at $\alpha=3.2$

\section{Conclusions}

We discussed different methods for solving linear boundary value problems for Caputo fractional Volterra integro-differential equations, namely, variational iteration method and homotopy perturbation method. The example is presented to illustrate the accuracy of the present schemes of VIM and HPM. Comparisons of VIM and HPM with exact solution have been shown by graphs are plotted which show the efficiency of the methods.

Acknowledgements. The authors would like to thank the anonymous reviewers and the editor of this journal for their helpful comments and valuable suggestions which led to an improved presentation of this paper.

\section{REFERENCES}

[1] O. Abdulaziz, I. Hashim, S. Momani, Application of homotopy-perturbation method to fractional IVPs, J. Comput. Appli. Math. 216 (2008), 574-584.

[2] M. Bani Issa, A. Hamoud, K. Ghadle, Giniswamy, Hybrid method for solving nonlinear Volterra-Fredholm integro-differential equations, J. Math. Comput. Sci. 7 (2017), 625-641.

[3] P. Darania, K. Ivaz, Numerical solution of nonlinear Volterra-Fredholm integrodifferential equations, Comput. Math. Appl. 56 (2008), 2197-2209. 
S.M. Atshan, A.A. Hamoud - Approximate solutions of fourth-order ...

[4] G. Ebadi, M. Rahimi-Ardabili, S. Shahmorad, Numerical solution of the nonlinear Volterra integro-differential equations by the Tau method, Appl. Math. Comput. 188 (2007), 1580-1586.

[5] M. Ghasemi, M. kajani, E. Babolian, Application of He's homotopy perturbation method to nonlinear integro-differential equations, Appl. Math. Comput. 188 (2007), $538-548$.

[6] K.P. Ghadle, A.A. Hamoud, Study of the approximate solution of fuzzy VolterraFredholm integral equations by using (ADM), Elixir Appl. Math. 98 (2016), 4256742573.

[7] J.H. He, Variational iteration method - a kind of non-linear analytical technique: some examples, International Journal of Nonlinear Mechanics, 34 (1999), 699-708.

[8] J.H. He, Homotopy perturbation method: a new nonlinear analytic technique, Appl. Math. Comput. 135 (2003), 73-79.

[9] J.H. He, Homotopy perturbation technique, Computer Methods in Applied Mechanics and Engineering, 178 (1999), 257-262.

[10] J.H. He, A coupling method of homotopy technique and perturbation technique for nonlinear problems, International Journal of Nonlinear Mechanics, 35, (1) (2000), $37-43$.

[11] A.A. Hamoud, K.P. Ghadle, On the numerical solution of nonlinear VolterraFredholm integral equations by variational iteration method, Int. J. Adv. Sci. Tech. Research, 3 (2016), 45-51.

[12] A.A. Hamoud, K.P. Ghadle, The combined modified Laplace with Adomian decomposition method for solving the nonlinear Volterra-Fredholm integro-differential equations, J. Korean Soc. Ind. Appl. Math. 21 (2017), 17-28.

[13] A.A. Hamoud, K.P. Ghadle, Modified Adomian decomposition method for solving fuzzy Volterra-Fredholm integral equations, J. Indian Math. Soc. 85, (1-2) (2018), 53-69.

[14] A.A. Hamoud, A.D. Azeez, K.P. Ghadle, A study of some iterative methods for solving fuzzy Volterra-Fredholm integral equations, Indonesian J. Elec. Eng. \& Comp. Sci. 11, (3) (2018), 1228-1235.

[15] A.A. Hamoud, K.P. Ghadle, The reliable modified of Laplace Adomian decomposition method to solve nonlinear interval Volterra-Fredholm integral equations, Korean J. Math., 25, (3) (2017), 323-334.

[16] A.A. Hamoud, K.P. Ghadle, Existence and uniqueness of solutions for fractional mixed Volterra-Fredholm integro-differential equations, Indian J. Math., 60, (3) (2018), (to appear). 
S.M. Atshan, A.A. Hamoud - Approximate solutions of fourth-order ...

[17] A.A. Hamoud, K.P. Ghadle, M.SH.Bani Issa, Giniswamy, Existence and uniqueness theorems for fractional Volterra-Fredholm integro-differential equations, Int. J. Appl. Math. 31, (3) (2018), 333-348.

[18] A.A. Hamoud, K.P. Ghadle, The approximate solutions of fractional VolterraFredholm integro-differential equations by using analytical techniques, Probl. Anal. Issues Anal. 7(25), (1) (2018), 41-58.

[19] S.J. Liao, An approximate solution technique not depending on small parameters: A special example, International Journal of Non-Linear Mechanics, 30, (3) (1995), 371-380.

[20] K. Maleknejad, Y. Mahmoudi, Taylor polynomial solution of high-order nonlinear Volterra-Fredholm integro-differential equations, Appl. Math. Comput., 145 (2003), 641-653.

[21] S. Samko, A. Kilbas and O. Marichev, Fractional Integrals and Derivatives, Theory and Applications, Gordon and Breach, Yverdon, 1993.

[22] N.H. Sweilam, Fourth order integro-differential equations using variational iteration method, Comput. Math. Appli. 54 (2007), 1086-1091.

[23] A. Yildirim, Solution of BVPs for fourth-order integro-differentials by using homotopy perturbation method, Comput. Math. Appli. 56 (2008), 3175-3180.

Shakir M. Atshan

Department of Mathematics,

Dhi Qar University,

Dhi Qar, Iraq.

email:s11h32@yahoo.com

Ahmed A. Hamoud

Department of Mathematics,

Taiz University,

Taiz, 96704, Yemen.

email: drahmedselwi985@gmail.com 